\title{
Performance of MIMO with Circular Antenna Array Using Correlation Matrix
}

\author{
LiJun Dong ${ }^{\dagger}$, MengWei Huang ${ }^{\dagger}$, Jie.Zhou $^{\dagger}$ and Hisakazu.Kikuchi ${ }^{\ddagger}$ \\ ${ }^{\dagger}$ Dept. of Information and Communication, \\ Nanjing University of Information Science and Technology, \\ Nanjing,China \\ ${ }^{\ddagger}$ Dept. of Electrical and Electronic Engineering, \\ Niigata University, 950-2181, Japan \\ dreamdlj@hotmail.com
}

\begin{abstract}
Spatial correlation is one of the impairments practical Multiple-Input Multiple-Output (MIMO) wireless communication systems have to be coped with. Capacity increases promised by MIMO systems mostly depend on the spatial correlation properties of the radio channels. This paper investigates the connection between these properties and the channel capacity. By investigating the channel capacity using the correlation matrix for a circular array, we prove that, for circular array, decrease in the radius is equivalent to decrease in signal to noise ratio $(S N R)$.
\end{abstract}

Keywords-MIMO, circular array, correlation, channel capacity, exact and approximate analysis

\section{INTRODUCTION}

Multiple-Input Multiple-Output (MIMO) techniques have recently emerged as a new paradigm. It has been shown that MIMO is a promising approach that can lead to very large bandwidth efficiency[1][2][3]. This spectral efficiency is achieved by transmitting different signals on different antenna simultaneously in the same frequency channel as well as using multiple receive antennas and the appropriate receive algorithms[4][5].

The spectral efficiency that can be exploited in MIMO depends on a number of phenomenons, including the average received power of desire signal, distribution of the received signal, thermal and implementation related noise, as well as interference generated by other users. Because of the multiple transmit antennas and multiple receive antennas, the multiple dimensional statistical characteristic is of foremost significance to the system. Then it is crucial for the system designers of a MIMO communication to have a spatial correlation model that appropriately presents the essential propagation characteristics $[6][7]$.

Numerous works published recently have quantified the potential benefits of such MIMO systems, considering various types of antenna set-ups placed in a variety of environments, see for instance Refs. [4] and [5]. In these literatures, various signal models that include spatial correlation have been proposed for the linear antenna array. Exponential correlation matrix model is also proposed for MIMO sys- tem because of its simplness, although it may be not an accurate model for real-world scenarios. To our knowledge, however these literatures are still lacking a simple but accurate correlation models from an uncorrelated to a fully correlated scenario. In this paper, the goal is to introduce a simple correlation model [6][7] for MIMO system in which the circular antenna array is considered, that is our main contribution. By investigating the MIMO channel capacity using the correlation matrix for a circular array, we prove that, for circular array, decrease in the radius is equivalent to decrease in signal to noise ratio (SNR).

\section{CORRELATION FORMULATION FOR CIRCULAR ARRAY}

Figure 1 shows the antenna array geometry used in our investigation where we have estimated a circular geometry with a radius $R$ and different antenna element $M$ where the antenna element $M$ lies about at a radius of $r=R$ for our circular antenna array. The multiple antennas may be omni-directional or have a non-uniform sensitivity to the angle and frequency signature of the incident waveform. The antennas may be equally or unequally spaced across the array.

Here we employ the spatial correlation models presented in the exact and approximate analysis. Considering two kinds of incident signal energy distributions, those are uniform distribution and Gaussian distribution.

\section{A. Gaussian Distribution}

Gaussian distribution can be represented as[5][7]

$$
\begin{aligned}
& p(\theta)=\frac{\kappa}{\sqrt{2 \pi} \sigma} e^{-(\theta-\phi)^{2} / 2 \sigma^{2}} \quad \text { for } \theta \in[-\pi+\phi, \pi+\phi] \\
& \text { and } \kappa=\frac{1}{\operatorname{erf}\left(\frac{\pi}{\sqrt{2} \sigma}\right)}
\end{aligned}
$$

where $\phi$ and $\sigma$ are the mean direction of arrival and the standard deviation of the distribution. $\kappa$ is the normalization factor, to make $p(\phi)$ a physical density function.where $\operatorname{erf}(x)=\frac{2}{\sqrt{\pi}} \int_{0}^{x} e^{-t^{2}} d t$ is the error function. 


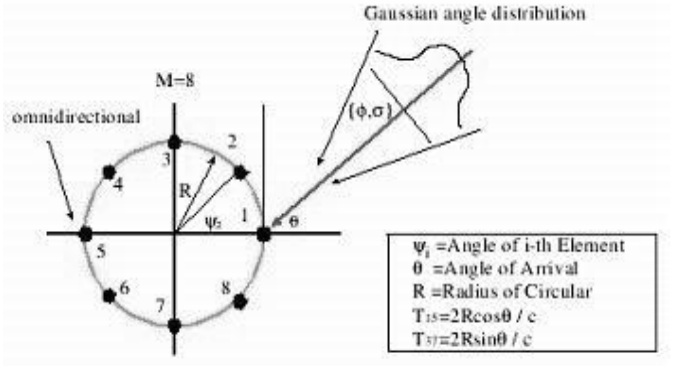

Fig. 1 Signal angle determination in a circular antenna array with 8 elements

According to Ref.[7], the correlation between m-th and $\mathrm{n}$-th elements by exact analysis is

$$
\begin{aligned}
& \operatorname{Re}[\rho(m, n)]= \frac{\kappa}{\sqrt{\pi}} \int_{-\frac{\pi}{\sqrt{2} \sigma}}^{\frac{\pi}{\sqrt{2} \sigma}} e^{-y^{2}}\left[J_{0}\left(Z_{C}\right)+\right. \\
&\left.2 \sum_{k=1}^{\infty} J_{2 k}\left(Z_{C}\right) \cos (2 k(\gamma+\sqrt{2} \sigma y+\phi))\right] d y \\
& \operatorname{Im}[\rho(m, n)]= \frac{\kappa}{\sqrt{\pi}} \int_{-\frac{\pi}{\sqrt{2} \sigma}}^{\frac{\pi}{\sqrt{2} \sigma}} e^{-y^{2}}\left[2 \sum_{k=0}^{\infty} J_{2 k+1}\left(Z_{C}\right)\right. \\
&\cdot \sin ((2 k+1)(\gamma+\sqrt{2} \sigma y+\phi))] d y
\end{aligned}
$$

For lower or intermediate $\sigma$, the approximate $\sin (z \sigma) \approx z \sigma$ and $\cos (z \sigma) \approx 1$ that gives the approximate equation as[7]

$$
\rho(m, n) \approx \kappa e^{-j Z_{C} \sin (\gamma+\theta)} e^{-\frac{\left(Z_{C}\right) \sigma \cos (\phi+\gamma)^{2}}{2}}
$$

here, $Z_{C}=\sqrt{Z_{1}^{2}+Z_{2}^{2}}$ in which $Z_{1}=2 \pi \frac{R}{\lambda}\left[\cos \left(\Psi_{m}\right)-\right.$ $\left.\cos \left(\Psi_{n}\right)\right]$ and $Z_{2}=2 \pi \frac{R}{\lambda}\left[\sin \left(\Psi_{m}\right)-\sin \left(\Psi_{n}\right)\right], \sin (\gamma)=$ $Z_{1} / Z_{C}$ and $\cos (\gamma)=Z_{2} / Z_{C}$ are defined.

\section{B. Uniform Distribution}

Another common assumption for angular energy distribution is a uniform distribution. The uniform distribution is defined as $[6]$

$$
p(\theta)=\frac{1}{2 \triangle}, \text { for } \theta \in[\phi-\triangle, \phi+\triangle]
$$

where $2 \Delta$ is the range of angles about a central angle-ofarrival $\phi$.

From Ref.[7], the real and imaginary parts of the correlation function $\rho(m, n)$ of circular antenna arrays for a uniform distribution can be expressed as

$$
\begin{aligned}
\operatorname{Re}[\rho(m, n)]= & J_{0}\left(Z_{C}\right)+2 \sum_{k=1}^{\infty} J_{2 k}\left(Z_{C}\right) \cos (2 k(\phi+\gamma)) \\
& \cdot \operatorname{sinc}(2 k \triangle)
\end{aligned}
$$
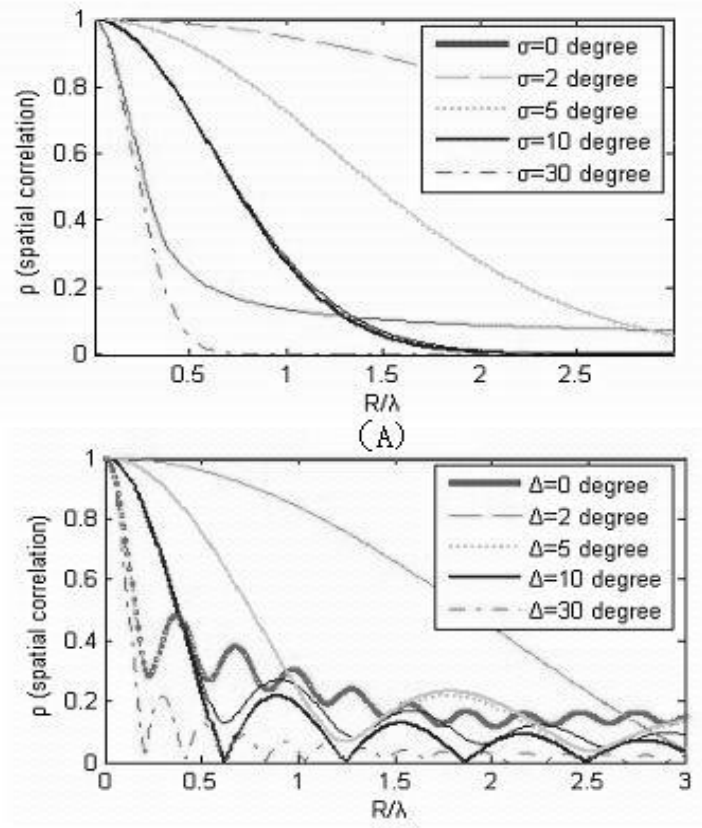

(B)

Fig. 2 Spatial correlation between the elements $1\left(\Psi_{1}=0\right.$ degree $)$ and $4\left(\Psi_{4}=135\right.$ degrees) versus $R / \lambda$, (A) uniform distribution of angular energy, (B) Gaussian distribution of angular energy.

$$
\begin{aligned}
\operatorname{Im}[\rho(m, n)]= & 2 \sum_{k=0}^{\infty} J_{2 k+1}\left(Z_{C}\right) \sin ((2 k+1)(\phi+\gamma)) \\
& \cdot \operatorname{sinc}((2 k+1) \triangle)
\end{aligned}
$$

From Ref.[7] as lower and intermediate $\Delta$, the approximate equation is

$$
\rho(m, n) \approx e^{-j Z_{C} \sin (\gamma+\theta)} \operatorname{sinc}\left[\left(Z_{C}\right) \Delta \cos (\phi+\gamma)\right]
$$

Comparing the exact analysis with the approximate analysis, the approximate equations can be simply calculated for low and moderate angle spread $\Delta$ and standard deviation $\sigma$, Ref.[7] concluded that the approximate analysis exhibits good agreement with the exact analysis when $\Delta<10$ degrees and $\sigma<10$ without the limitation of $d / \lambda$ and $R / \lambda$. One also can see the Gaussian distribution decreases more slowly in the main lobe, but lacks the secondary correlation peaks contract with uniform distribution. $R / \lambda<0.25$ for the circular antenna array and angle spread $\Delta<30$ degrees and standard deviation $\sigma<30$ degrees, the approximate analysis can be used to instead of exact analysis during simulating the antenna array. All the numerical results can be seen in the Fig.2.

\section{MIMO PERFORMANCE}

\section{A. MIMO System}


MIMO communication systems can significantly increase the data rate of wireless system without increasing transmitting power and allocated bandwidth. Figure 2 presents a MIMO system employing $N t$ transmit $(T x)$ and $N r$ receive $(R x)$ antennas. The transmitter sends an $N t$ dimensional complex signal vector $s$. The receiver records an $N r$ dimensional complex vector $\boldsymbol{x}$ which depends on $\boldsymbol{s}$ via[4][5]

$$
X=\mathbf{H} \cdot \mathbf{s}+\mathbf{n}
$$

where $\boldsymbol{H}$ is an $N r^{*} N r$ complex propagation matrix that is constant per packet transmission and assumed to be known at the receiver. Element $(n, m)$ of $\mathbf{H}$ contains the normalized flat-fading channel response from $T x$ antenna $\mathrm{m}$ to $R x$ antenna $\mathrm{n}$ with variance $\sigma_{c}^{2}=1$. The vector $\mathbf{n}$ is $\mathrm{Nr}$ dimensional and represents zero means, complex additive white Gaussian noise (AWGN) with covariance matrix $E\left[\mathbf{n} \cdot \mathbf{n}^{T}\right]=\sigma_{n}^{2} \mathbf{I}$, where $(\cdot)^{T}$ denotes the conjugate transpose of the corresponding vector or matrix and $\mathbf{I}$ represents the identity matrix, here having dimension $N r$. The total power of s is $P$, i.e. independent of number $T x$ antennas.

Without loss of generality, we assume $N r=N t=N$ for simplification in this paper, i.e. the MIMO system has $N$ transmit antennas and also $N$ receive antennas. For a fixed linear $N * N$ matrix channel with AWGN, the MIMO channel capacity is[4][5]

$$
C=B \cdot \log _{2}\left|I+\frac{\gamma}{N} \mathbf{H} \cdot \mathbf{H}^{T}\right|
$$

when the transmitted signal vector is composed of statistically independent equal power components each with a Gaussian distribution and the receiver knows the channel. $\gamma$ is the average signal-to-noise $(S N R)$, and $\gamma / N$ is the $S N R$ per receive branch. As described above, $\mathbf{H}$ is an $N^{*} N$ complex propagation matrix under flat-fading, and its components satisfy the following normalization condition[3]

$$
\sum_{i=1}^{N} \sum_{j=1}^{N}\left|h_{i j}\right|=N
$$

In the case of all parallel sub-channels are independent that is $\mathbf{H}=\mathbf{I}$, the system achieves its maximum capacity as $C=N B \log _{2}\left(1+\frac{\gamma}{N}\right)$.

\section{B. Channel Capacity}

In practice, the channel is random and then the channel capacity is also random in this case we need to consider its average means or its ergodic capacity as follows[4][5]

$$
C=E\left[B \cdot \log _{2}\left|I+\frac{\gamma}{N} \mathbf{H} \cdot \mathbf{H}^{T}\right|\right]
$$

According to Jensen inequality and the capacity of $\log _{2}|\cdot|$, the average capacity in Eq.(10) can be bounded by

$$
C \leqq B \cdot \log _{2}\left|I+\frac{\gamma}{N} R\right|
$$

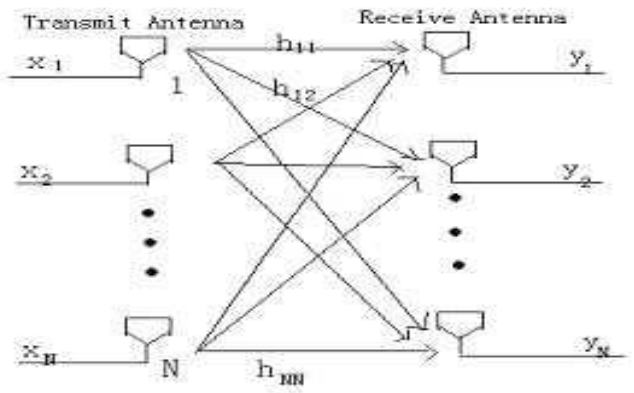

Fig. 3 MIMO system with $N$ transmit and $N$ receive antenna elements

where,

$$
R=E\left[\mathbf{H} \cdot \mathbf{H}^{T}\right]
$$

and its element $\gamma_{i j}$ in $\mathrm{R}$ matrix is equal to

$$
\gamma_{i j}=\sum_{k=1}^{N} E\left[h_{i j} \cdot h_{i j}^{*}\right]
$$

where $*$ presents complex conjugate. To study the effects of correlation on an exploit method, we separate it from the effects of unequal receive powers by assuming as follows

$$
\sigma_{i}=\sum_{j=1}^{N}\left|h_{i j}\right|^{2}=\sum_{j=1}^{N} h_{i j} \cdot h_{i j}^{*}=1
$$

In other words, all receive powers in $N$ receive antennas are equal. With the assumption, the normalized correlation coefficient $\gamma h_{i j}$ is defined as

$$
\rho_{i j}=\frac{1}{\sqrt{\sigma_{i} \cdot \sigma_{j}}}=\gamma_{i j}
$$

Equation(15) presents that $\gamma_{i j}$ is the normalized correlation coefficient and that $R$ is the normalized channel correlation matrix. Then Equation(11) denotes the correlated fading effects on MIMO channel capacity. In our practical situation, the normalized correlation coefficient $\rho_{i j}$ is the $\rho(m, n)$ described in section II for our circular antenna array. After some derivations, Equation(11) can also be rewritten into the following formula as

$$
C \leqq C \max +B \cdot \log _{2}|\Lambda|
$$

$$
\Lambda=\left(\begin{array}{ccccc}
1 & B \rho_{12} & B \rho_{13} & \ldots & B \rho_{1 M} \\
B \rho_{12}^{*} & 1 & B \rho_{23} & \ldots & B \rho_{2 M} \\
\vdots & \vdots & \vdots & \vdots & \vdots \\
B \rho_{1 M}^{*} & B \rho_{2 M}^{*} & B \rho_{3 M}^{*} & \ldots & 1
\end{array}\right)_{M \times M}
$$

where,

$$
\beta=\frac{\gamma}{N}\left(1+\frac{\gamma}{N}\right)^{-1}
$$


Here, $\rho_{i j}$ is referred as correlation coefficients and the asterisk indicates the complex conjugate. Here, the correlation coefficients are just the analytical formulas in Sect.II as $\rho_{i j}=\sqrt{\operatorname{Re}^{2}[\rho(m, n)]+\operatorname{Im}^{2}[\rho(m, n)]}$. The channel capacity formula (16) directly presents that correlation fading reduces the capacity as $0 \leqq|\Lambda| \leqq 1$. In Sect.II three different correlation models are used to determine the correlation matrix, then we can fully analyze the effects of correlation, aperture size of antenna array and the number of antenna elements on the channel capacity. Also we can compare our numerical results with that of exponential correlation matrix model[5].

In above sections, we derived the correlation formula and channel capacity. From Eqs.(11)and(12), the capacity $C$ of the average channel provides an upper bound on the mean (or ergodic) capacity. To prove the above derivations, the capacity $C$ of the average can be evaluated numerical by Matlab Program. In order to estimate the mean capacity by simulation, the following assumptions are used as

(1)When considering multiple paths, assuming that there are $\mathrm{n}$ multiple paths arriving to each receive antenna element from a transmit antenna.

(2)For Gaussian distribution, the angles of arriving (AOA) of these paths are random distributed with Gaussian variables by standard deviation $\sigma$. To uniform distribution, the AOA of these paths are uniform distributed in $[-\Delta,+\Delta]$.

(3) Rayleigh fading is considering in the gains of these multiple paths that is with zero mean and unit variance.

(4) Each transmit antenna transmits an independent se of $n$ paths with the same statistical characteristics as $\operatorname{in}(3)$.

According to above assumptions, all the terms in Eq.(13) can be calculated and programmed. Then we can evaluate the average channel capacity by Matlab Program for different $\sigma, \Delta$, and antenna array radius $\mathrm{R}$. In the following section, we present the numerical and simulating results for the circular antenna array.

\section{NUMERICAL RESULTS}

From the analysis of the last section we can derive the MIMO capacity of a deterministic channel versus the correlation coefficient evaluated by the exact and approximate analysis. In this section we analysis the different performances of the MIMO capacity with circular array using correlation when considering the different parameters $\sigma(\Delta)$, $\mathrm{R}$ and $\mathrm{n}$. The calculation of the spatial correlation is under the distribution of angular energy. Here considering two kinds of incident signal energy distributions, those are uniform distribution and Gaussian distribution.

From the Fig.4, the conclusion is derived that with the increase of the angle spread $\Delta$ and standard deviation $\sigma$, the capacity of the circular MIMO system becomes higher. When $\sigma$ and $\Delta$ change from 5 degrees to 10 degrees, the capacity increases by more than $30 \%$. Then the range of

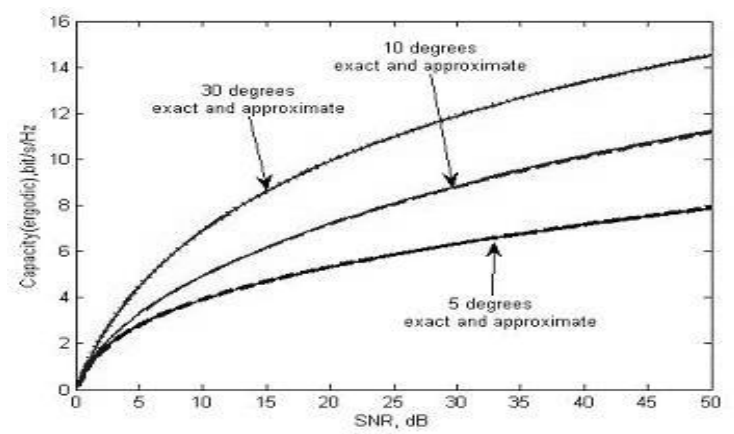

(a)

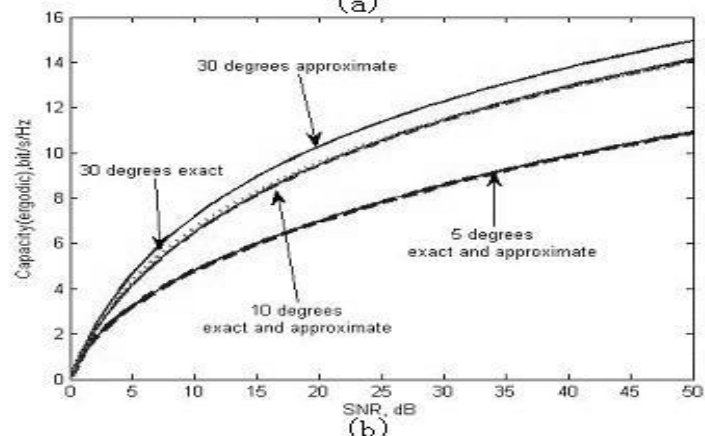

(b)

Fig. 4 MIMO channel capacity versus SNR with different $\sigma$ and $\Delta$ when $n=4, R / \lambda=0.5$, (a) Gaussian distribution of angular energy ( $\phi=30$ degrees, $\xi=0$ degrees), (b) Uniform distribution of angular energy ( $\phi=30$ degrees, $\xi=0$ degrees)
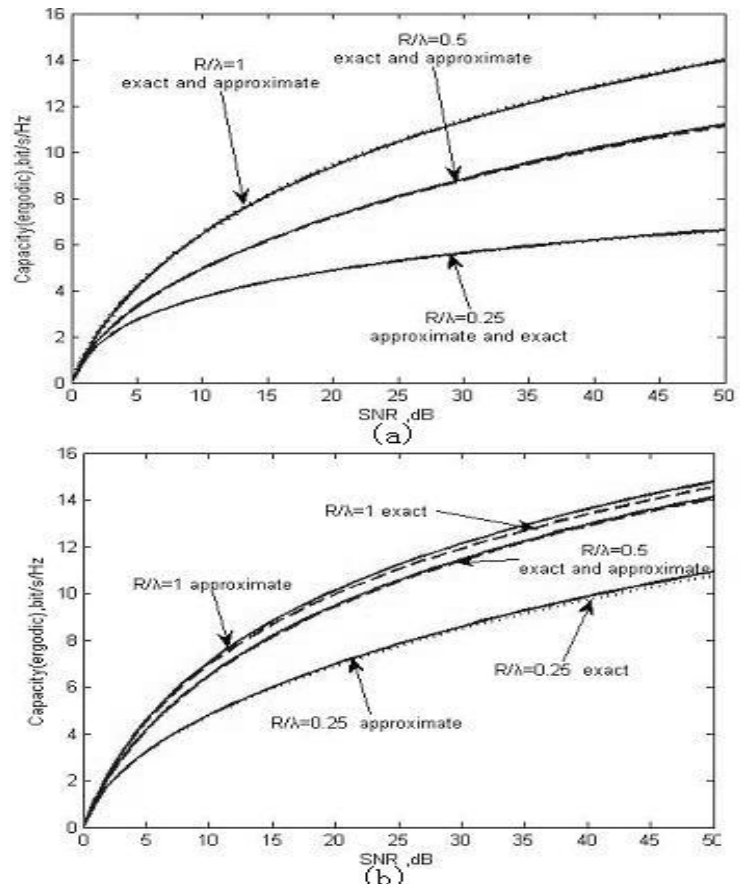

Fig. 5 MIMO channel capacity versus SNR with different $R / \lambda$ when $n=4$ and $\sigma=\Delta=10$ degrees, (a) Gaussian distribution of angular energy ( $\phi=30$ degrees, $\xi=0$ degrees), (b) Uniform distribution of angular energy ( $\phi=30$ degrees, $\xi=0$ degrees) 

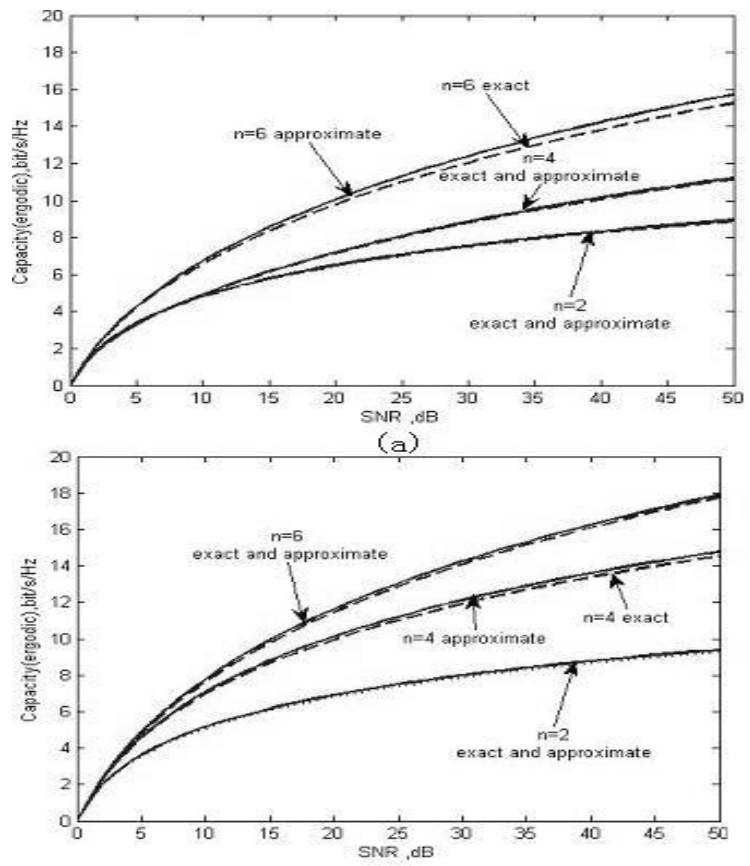

(b)

Fig. 6 MIMO channel capacity versus $S N R$ with different $n$ when $R / \lambda=1$ and $\sigma=\Delta=10$ degrees, (a) Gaussian distribution of angular energy ( $\phi=30$ degrees, $\xi=0$ degrees), (b) Uniform distribution of angular energy ( $\phi=30$ degrees, $\xi=0$ degrees)

the AOA has an apparent influence on the capacity of the system. The approximate analysis exhibits good agreement with the exact analysis when $\Delta<10$ degrees and $\sigma<10$ without the limitation of $d / \lambda$ and $R / \lambda$.

As we see in Fig.5, the parameter $R / \lambda$ is also important for the analysis of the system capacity. It is obvious that the increase of $R / \lambda$ means the decrease of the correlation of antennas. When the $R / \lambda$ is big enough, the correlation gets close to zero and the capacity must to be the best. And from the figure, we can also get the conclusion that under the Gaussian distribution the system has better performance compared with the situation under uniform distribution.

Ideally, the more antenna is, the higher the system capacity become. However from Fig. 6 we get the conclusion that with a certain $R$, the increase of the antenna number means the reduction of the antenna distance, which leads to the addition of correlation.

Obviously the correlation model has very good performance when studying the performance of the circular antenna array. It is seen that approximate analysis has better fit with the accurate analysis when Gaussian distribution of incident signal energy is considered.

It is noticed that with the increase of the parameters $\sigma(\Delta), R / \lambda$ and the number of antenna $\mathrm{n}$, the MIMO capacity steps up and the curves rise more rapidly when the pa- rameters increase. Comparing the uniform angular energy distribution with the Gaussian distribution, higher capacity is predicted.

The correlation model introduced here has very good performances in computational efficiency. From the figures we know that for lower and intermediate $\sigma(\Delta)$, the approximate equations fit well with the exact equations. However the approximate equations saved about $50 \%-70 \%$ computational time compared with the exact equations. In practical computation this model can greatly minish operation time and difficulty compared with other models.

\section{CONCLUSIONS}

The capacity of the MIMO systems depends substantially on the special correlation properties of the radio channels. This paper investigates the connection between these properties and the channel capacity. We derived generalized spatial correlation functions for a circular antenna array. These equations are investigated carefully by exact and approximate analysis. The generalized formulas allow the correlation to be found for any practical standard deviation and antenna array geometry. By investigating the channel capacity using the correlation matrix for a circular array, we prove that, for circular array, decrease in the radius is equivalent to decrease in signal to noise ratio $(S N R)$.In this paper, a simple correlation model is introduced for MIMO system in which the circular antenna array is considered. And the model has a good performance while studying the circular antenna array. The biggest advantage of the approximate formulas lies in the good fitness with the exact ones as well as the saving of computational time.

\section{References}

[1] J.G.Proakis, Digital communications, McGraw-Hill Higher Education, Thomas Casson, 2000.

[2] A.F.Naguib, "Adaptive antenna for CDMA wireless network," $\mathrm{PhD}$ thesis, Standford University, Palo Alto, CA, Aug. 1996

[3] P.D. Teal, T.D. Abhayapala and R.A. Kennedy, "Spatial correlation in non-isotropic scattering scenarios," Proc. IEEEVeh. Technol. Conference (VTC'98), PP.489-493,May 1998

[4] Y.K. Cbo and J.H. Lee, "Effect of fading correlation on the SER performance of M-ary PSK with maximal ratio combining," IEEE Trans. on Communication Letters, Vol.3,No.7,pp.199201,July 1999

[5] S.L. Loyka, "Channel capacity of MIMO architecture using exponential correlation matrix," IDEE Trans.on Communication Letters. Vol.5,NO.9 pp.369-372, Sept.2001

[6] J.A.Tsai and B.D.Woerner, "The fading correlation function of a circular antenna array in mobile radio environment," Proc. IEEE Global Telecommunications Conference (Globecom'01), San Antonio, Texas, USA, Nov. 2001.

[7] J.Zhou, K.Isgizawa, S.Sasaki, H.Kikuch, "Generalized spatial correlation equations for antenna arrays in wireless diversity reception: Exact and Approximate Analysis," IEICE Trans.On Communication.Vol.E87-B,No.1,pp.204-210,Jan.2004 